\title{
Thoracoscopic-Assisted Diaphragmatic Hernia Repair Using a Thoracic Rib Resection
}

\author{
ERIN D. MALONE, DVM, PhD, Diplomate ACVs, KELLY FARNSWORTH, DVM, MS, TIFFANY LENNOX, DVM, \\ JULIA TOMLINSON, BVSc, MRCVS, and ABBY M. SAGE, VMD, MS, Diplomate, ACVIM
}

\begin{abstract}
Objective-To describe the surgical treatment of a dorsal diaphragmatic hernia in an adult horse using thoracic rib resection aided by thoracoscopy and a flank incision.

Study Design-Case report.

Animal Population-One client-owned horse.

Results-A six-year-old Dutch warmblood gelding was admitted for colic-associated colonic incarceration in a diaphragmatic hernia. Seven weeks after the initial colic surgery, the gelding underwent surgery to repair the defect. Thoracoscopy and a flank incision were used to identify the location of the hernia and the subsequent site of rib resection. The stomach was adhered to the edges of the defect, leaving only a small residual hole. Resection of the 14th rib provided excellent exposure of the defect and closure of the remaining hernia.

Conclusion and Clinical Relevance-Thoracic rib resection can provide access to diaphragmatic hernias in adult horses. Thoracoscopy or a flank incision, or both, may aid in determining which rib is best resected.

(C) Copyright 2001 by The American College of Veterinary Surgeons
\end{abstract}

$\mathrm{T}$ HE PROGNOSIS for diaphragmatic hernias in adult horses is guarded because of limited access to the defects. ${ }^{1-4}$ Many of the defects require mesh implantation because of the size of the rent and the friable nature of the tissue..$^{2-5}$ Often, repair is attempted by ventral median celiotomy, with limited to no visualization of the surgical site and hindrance of the procedure by abdominal viscera. ${ }^{4-6}$ These horses are also usually at greater anesthetic risk because of respiratory compromise from the lesion, and may already be undergoing prolonged surgical procedures even without the addition of a complicated hernia repair. ${ }^{7}$ This report describes the successful delayed repair of a diaphragmatic hernia using a lateral approach and a thoracic rib resection in an adult warmblood gelding.

\section{CASE REPORT}

A 6-year-old, 557-kg, Dutch warmblood gelding was admitted for mild signs of colic of 18 hours duration after treatment with intragastric mineral oil and intravenous analgesics (xylazine, butorphanol, and flunixin meglumine). Purchased as a 5-year-old, there was no known history of colic, exercise intolerance, or significant trauma.

On initial examination, the gelding was showing mild signs of colic and appeared depressed, with a heart rate of 60 beats per minute and a rectal temperature of $99.4^{\circ} \mathrm{F}$. His respiratory rate was 30 breaths per minute and labored, with nostril flaring and abdominal press noted; lung sounds were normal. Gastrointestinal sounds were noted to be slightly more cranial than

From the Department of Clinical and Population Sciences, University of Minnesota Veterinary Teaching Hospital, St. Paul, MN. Address reprint requests to Erin Malone, DVM, PhD, Department CAPS, 225K VTH, 1365 Gortner Ave, St. Paul, MN 55108. (C) Copyright 2001 by The American College of Veterinary Surgeons 0161-3499/01/3002-0010\$35.00/0 doi:10.1053/jvet.2001.21395 
expected. He was moderately dehydrated (packed cell volume, $40 \%$; total plasma protein, $8.8 \mathrm{~g} / \mathrm{dL}$ ), but a complete blood count and serum biochemical analyses were unremarkable. Fluid obtained from abdominocentesis was normal. No reflux was obtained on nasogastric intubation. On rectal examination, no manure was found in the rectum and the pelvic flexure was not palpable. No distended bowel or tight bands were palpable.

Diaphragmatic hernia was suspected based on the labored breathing, cranial gastrointestinal sounds, and lack of abdominal viscera on rectal palpation. Ultrasound examination of the thorax and abdomen revealed aerated pulmonary parenchyma in the dorsal left hemithorax and a gas-filled viscus in the ventral left hemithorax that was most compatible with large intestine. The diaphragm was observed ventral to the large intestine. Pleural effusion was present in the ventral right thoracic cavity. Aerated pulmonary parenchyma was observed in the dorsal right hemithorax. On thoracic radiographs, there was displacement of the ascending colon into the thoracic cavity, as well as pulmonary consolidation and pleural fluid.

\section{SURGICAL PROCEDURE}

Because of the extent of herniation and the continued signs of colic, an exploratory celiotomy was performed. Intranasal oxygen was administered preoperatively, induction was performed with guaifenesin and ketamine, and anesthesia was maintained with isoflurane in oxygen using positive-pressure ventilation. Dobutamine and ephedrine were administered to control hypotension. A standard ventral median incision was performed, extended cranially toward the xiphoid cartilage. A few feet of small intestine and over $75 \%$ of the ascending colon was found to be herniated through a $15-\mathrm{cm}$ defect located approximately $5 \mathrm{~cm}$ ventral to the dorsal attachment of the diaphragm, just to the left of midline. The rent had smooth, fibrous edges and was resistant to stretch. The ascending colon was positioned along the inner surface of the diaphragm, with the pelvic flexure close to the xiphoid. The small intestine was easily removed; however, attempts made to free the colon using manipulation and gas decompression through the diaphragm were unsuccessful. A small opening was made in the middle of the diaphragm using digital dissection, after which the colon was easily decompressed and could be removed from the thoracic cavity. The small diaphragmatic hole was closed with 1 polydioxanone using a cruciate pattern. After removal of the colon, the stomach and a portion of liver settled over the defect, making it difficult to relocate. The colon appeared relatively undamaged, with serosal hemorrhage as the only significant lesion. No other significant findings were detected on abdominal exploration.

Options previously discussed with the owner included colonopexy and attempts to close the hernia at the time of surgery. However, the gelding's condition was deteriorating under anesthesia, and any repair attempts were deemed too dangerous to pursue at that time. The abdominal incision was closed and air evacuated from both the thoracic and abdominal cavities. Anesthetic recovery was uneventful. After recovery, $2 \mathrm{~L}$ of hemorrhagic fluid was removed from the left thorax. A thoracic drain was maintained for 14 hours. Repeat ultrasound the day after surgery revealed bilateral pleural effusion, pneumothorax, and pneumoperitoneum. No abdominal contents were observed within the thorax. The horse was maintained on intravenous (IV) potassium penicillin $(22,000 \mathrm{U} / \mathrm{kg}$ every 6 hours) and gentamicin $(6.6 \mathrm{mg} / \mathrm{kg}$ IV once daily) for 4 days. Heparin (40,000 U/kg subcutaneously every 8 hours) was given for 2 days and decreasing dosages of flunixin meglumine IV for 4 days. No major postoperative complications developed, and the horse was discharged 5 days after surgery.

Seven weeks after the initial surgery, the gelding was readmitted for hernia repair. Repeat physical examination and thoracic ultrasound revealed no significant abnormalities. The diaphragmatic hernia could not be observed ultrasonographically, but following the line of the ventrolateral diaphragmatic crus suggested that the defect would be under the 15th or 16th rib. A decision was made to try a lateral approach using a thoracic rib resection, but to be prepared to enter the abdomen ventrally if this proved unsuccessful. Additionally, thoracoscopy was to be used to observe the defect and to most accurately determine which rib would require resection. The horse was administered preoperative potassium penicillin, gentamicin, and flunixin meglumine as described before. After induction of anesthesia, the horse was positioned in right lateral recumbency and maintained with isoflurane using positive-pressure ventilation. A $10-\mathrm{mm} 0^{\circ}$ rigid endoscope (Richard Wolf Medical Instruments Corporation, Vernon Hills, IL) was inserted cranial to the 15 th rib on the left side. The 
diaphragm and lung could be observed, but no defect could be identified. The endoscope was reinserted at the 17 th rib, with the same results. A $15-\mathrm{cm}$ flank incision was made caudal to the last rib to enable palpation of the area. This revealed that the stomach had adhered to the defect, leaving only a $2.5-\mathrm{cm}$ diameter hernia at the ventral aspect. With digital manipulation, this defect could be seen with the endoscope, but otherwise, it layed flat and appeared as continuous diaphragm. A $25-\mathrm{cm}$ section of the 14th rib was removed and the thoracic cavity opened. This provided an excellent view of the defect. The caudal edges of the defect were freshened slightly. The stomach and liver comprised the other margins. The edge of the diaphragm was sutured to the stomach using preplaced vertical mattress sutures of 0 polydioxanone. After tightening the sutures, the area was repalpated using the flank incision to ensure no entrapment of other structures; then all incisions were closed. Air was removed from the thorax and a thoracic drain placed before recovery. Anesthetic recovery was uneventful, and the drain was removed after 2 hours.

The gelding recovered rapidly after surgery. Intravenous antibiotics were continued for 4 days, and phenylbutazone was continued for 2 days. Free-choice hay was offered the following morning. The gelding had minimal evidence of discomfort and was discharged 5 days' postoperatively. Eight months after the surgery, he had returned to jumping, with no evidence of colic or exercise intolerance.

\section{DISCUSSION}

Thoracoscopy combined with thoracic rib resection provided excellent access to a dorsal diaphragmatic hernia in an adult horse. Use of a flank incision substantially increased the diagnostic capability without increasing morbidity.

Several options were considered for treatment of the diaphragmatic defect in this horse. These options included: pursuing no further treatment, attempting primary closure, or attempting mesh application by a ventral celiotomy, flank incision, or abdominal or thoracic rib resection. Because of the location and relatively small size of the hole in this gelding, it was considered unlikely that the colon would reherniate; however, it was likely that the small intestine could pass into the thorax. Ideally, the stomach and liver would provide a patch for the hole, but the stability of such a patch was questionable. Because the gelding was used as a jumper, repair of the defect because of the risk of reherniation was recommended..$^{5,6}$ The use of mesh was considered likely to be necessary because of the fibrous border and subsequent lack of elasticity of the defect noted at the initial surgery. Laparoscopic evaluation was considered as a possible means of determining whether the stomach had sealed the hole, making further surgery unnecessary. Standing laparoscopic closure of the lesion would have avoided the risks of anesthesia, but tension pneumothorax was a concern.

Ventral celiotomy is the standard approach for equine diaphragmatic hernia repair, with repair performed blindly by hand or with a hand-held stapler. ${ }^{2,5,6}$ However, the deep chest in the warmblood breed and the dorsal location of the hernia may have made this approach problematic. Use of a thoracic rib resection provided excellent observation of the surgical field and would have allowed for closure of the defect with mesh, if needed. The lateral approach has been reported in cattle ${ }^{8,9}$ and in one horse ${ }^{10}$; that horse died 18 hours after the surgery. We found the approach to be straightforward and to cause minimal evidence of pain postoperatively. We did take precautions to ensure reinflation of the lungs before recovery and carefully monitored for pneumothorax during recovery, particularly because the uppermost and typically best-perfused lung may also have been compromised by the previous hernia or surgery, or both events.

Flank incisions are used more typically in standing procedures when cost or medical concerns prohibit general anesthesia. Exposure through a flank incision is restricted by the size of the paralumbar space and by the horses' low tolerance for pain associated with visceral manipulation. ${ }^{11}$ The flank incision in this horse was of adequate size to easily palpate the area, and was used to confirm that the defect was partially blocked by the stomach and that the adhesions were secure.

In this horse, both thoracoscopy and the flank incision were useful in optimizing the surgical approach. Based on preoperative planning, our incision would have been located more dorsocaudally than was ideal without the use of the thoracoscope. The flank incision allowed direct palpation of the defect, and enabled us to more accurately identify the site of the defect and to ensure that the stomach was securely adhered to the defect rather than being temporarily in 
that position. In many instances, thoracoscopy alone may be adequate to determine the lesion location and subsequent surgical approach. Alternately, the flank incision may be useful in identifying which rib should be resected, particularly when thoracoscopic equipment is not available. In some cases, the defect may be able to be closed by the same flank incision; however, many of the complications of the ventral approach (depth of lesion, poor exposure, and obscuring viscera) can limit the flank approach.

\section{REFERENCES}

1. Corley JR, Bertone A: Diaphragmatic hernia in a horse. Equine Pract 12:28-31, 1990

2. Hance SR, Clem MF, DeBowes RM, et al: Intraabdominal hernias in horses. Comp Contin Educ Pract Vet 13:293-299, 1991

3. Steenhaut M, Verschooten F, De Moor A, et al: Surgical correction of diaphragmatic hernia in the horse: A report of 6 cases. Diergeneeskd Tijdschr 61:181-186, 1992

4. Edwards GB: Diaphragmatic hernia-A diagnostic and surgical challenge. Equine Vet Educ 5:267-269, 1993

5. Dabareiner RM, White NA: Surgical repair of a diaphragmatic hernia in a racehorse. J Am Vet Med Assoc 214:1517-1518, 1999

6. Bristol DG: Diaphragmatic hernias in horses and cattle. Comp Contin Educ Pract Vet 8:S407-S412, 1986

7. Santschi EM, Juzwiak JS, Moll HD, et al: Diaphragmatic hernia repair in three young horses. Vet Surg 26:242-245, 1997

8. De Moor A, Verschooten F, Desmet P: Thoracic repair of a diaphragmatic hernia in a heifer. Vet Rec 85:87-89, 1969

9. Krishnamurthy D, Deshpande KS, Nigam JM, et al: Repair of diaphragmatic hernia in the bovine-transthoracic approach. Indian J Vet Surg 1:7-11, 1980

10. Mason TA, Johnston DE, Wallace CE, et al: Laparotomy in equine colic_A report of thirteen clinical cases. Aust Vet J 46:349-355, 1970

11. Ducharme NG, Freeman DE, Steckel RR, et al: Principles of intestinal surgery, in Auer JA (ed): Equine Surgery. Philadelphia, PA: Saunders, 1992, pp 325-348 